\title{
MULTIPLIER IDEALS OF SUMS VIA CELLULAR RESOLUTIONS
}

\author{
Shin-YaO Jow AND EzRA Miller
}

\begin{abstract}
Fix nonzero ideal sheaves $\mathfrak{a}_{1}, \ldots, \mathfrak{a}_{r}$ and $\mathfrak{b}$ on a normal $\mathbb{Q}$-Gorenstein complex variety $X$. For any positive real numbers $\alpha$ and $\beta$, we construct a resolution of the multiplier ideal $\mathcal{J}\left(\left(\mathfrak{a}_{1}+\cdots+\mathfrak{a}_{r}\right)^{\alpha} \mathfrak{b}^{\beta}\right)$ by sheaves that are direct sums of multiplier ideals $\mathcal{J}\left(\mathfrak{a}_{1}^{\lambda_{1}} \cdots \mathfrak{a}_{r}^{\lambda_{r}} \mathfrak{b}^{\beta}\right)$ for various $\lambda \in \mathbb{R}_{\geq 0}^{r}$ satisfying $\sum_{i=1}^{r} \lambda_{i}=\alpha$. The resolution is cellular, in the sense that its boundary maps are encoded by the algebraic chain complex of a regular CW-complex. The CW-complex is naturally expressed as a triangulation $\Delta$ of the simplex of nonnegative real vectors $\lambda \in \mathbb{R}^{r}$ with $\sum_{i=1}^{r} \lambda_{i}=\alpha$. The acyclicity of our resolution reduces to that of a cellular free resolution, supported on $\Delta$, of a related monomial ideal. Our resolution implies the multiplier ideal sum formula

$$
\mathcal{J}\left(X,\left(\mathfrak{a}_{1}+\cdots+\mathfrak{a}_{r}\right)^{\alpha} \mathfrak{b}^{\beta}\right)=\sum_{\lambda_{1}+\cdots+\lambda_{r}=\alpha} \mathcal{J}\left(X, \mathfrak{a}_{1}^{\lambda_{1}} \cdots \mathfrak{a}_{r}^{\lambda_{r}} \mathfrak{b}^{\beta}\right)
$$

generalizing Takagi's formula for two summands [Tak05], and recovering Howald's multiplier ideal formula for monomial ideals [How01] as a special case. Our resolution also yields a new exactness proof for the Skoda complex [Laz04, Section 9.6.C].
\end{abstract}

\section{Introduction}

Let $X$ be a smooth complex algebraic variety and let $\mathfrak{a} \subseteq \mathcal{O}_{X}$ be an ideal sheaf. Applications in algebraic geometry of the multiplier ideal sheaves

$$
\mathcal{J}\left(\mathfrak{a}^{\alpha}\right)=\mathcal{J}\left(X, \mathfrak{a}^{\alpha}\right) \subseteq \mathcal{O}_{X}
$$

for real numbers $\alpha>0$ have led to investigations of their behavior with respect to natural algebraic operations. For example, Demailly, Ein, and Lazarsfeld [DEL00] proved that given two ideal sheaves $\mathfrak{a}_{1}$ and $\mathfrak{a}_{2}$, one has

$$
\mathcal{J}\left(\left(\mathfrak{a}_{1} \mathfrak{a}_{2}\right)^{\alpha}\right) \subseteq \mathcal{J}\left(\mathfrak{a}_{1}^{\alpha}\right) \mathcal{J}\left(\mathfrak{a}_{2}^{\alpha}\right) .
$$

For the subtler case of sums, on the other hand, Mustaţă [Mus02] showed that

$$
\mathcal{J}\left(\left(\mathfrak{a}_{1}+\mathfrak{a}_{2}\right)^{\alpha}\right) \subseteq \sum_{0 \leq t \leq \alpha} \mathcal{J}\left(\mathfrak{a}_{1}^{\alpha-t}\right) \mathcal{J}\left(\mathfrak{a}_{2}^{t}\right),
$$

and Takagi [Tak05] later refined this to

$$
\mathcal{J}\left(\left(\mathfrak{a}_{1}+\mathfrak{a}_{2}\right)^{\alpha}\right)=\sum_{0 \leq t \leq \alpha} \mathcal{J}\left(\mathfrak{a}_{1}^{\alpha-t} \mathfrak{a}_{2}^{t}\right),
$$

where he proved it more generally when $X$ is normal and $\mathbb{Q}$-Gorenstein.

Takagi used characteristic $p$ methods to deduce (1), which makes his work distinctly different from [DEL00] and [Mus02], where the arguments proceed by geometric techniques such as log resolutions and sheaf cohomology. Our purpose is to show that

Received by the editors April 14, 2007.

Key words and phrases. Multiplier ideal, summation formula, cellular resolution, monomial ideal, homology-manifold-with-boundary. 
such geometric techniques, combined with combinatorial methods from topology and commutative algebra, can recover Takagi's equality (1) and generalize it. As a consequence, we derive a new proof (Corollary 3) of Howald's formula for multiplier ideals of monomial ideals [How01], and demonstrate how it can be reformulated to hold for all ideals. In addition, we obtain a new "cellular" exactness proof (Corollary 4.4) for the Skoda complex [Laz04, Section 9.6.C] via the contractibility of simplices. Our main results center around the following, which constitutes part of Theorem 3.6.

Theorem 1. Fix nonzero ideal sheaves $\mathfrak{a}_{1}, \ldots, \mathfrak{a}_{r}, \mathfrak{b}$ on a normal $\mathbb{Q}$-Gorenstein complex variety $X$ and $\alpha, \beta>0$. There is a resolution $0 \rightarrow \mathcal{J}_{r-1} \rightarrow \cdots \rightarrow \mathcal{J}_{0} \rightarrow 0$ of the multiplier ideal $\mathcal{J}\left(\left(\mathfrak{a}_{1}+\cdots+\mathfrak{a}_{r}\right)^{\alpha} \mathfrak{b}^{\beta}\right)$ by sheaves $\mathcal{J}_{i}$ that are finite direct sums of multiplier ideals of the form $\mathcal{J}\left(\mathfrak{a}_{1}^{\lambda_{1}} \cdots \mathfrak{a}_{r}^{\lambda_{r}} \mathfrak{b}^{\beta}\right)$ for various nonnegative $\lambda \in \mathbb{R}^{r}$ with $\sum_{i=1}^{r} \lambda_{i}=\alpha$. Every distinct ideal sheaf of that form appears as a summand of $\mathcal{J}_{0}$.

Part of the final claim of Theorem 1 is that there are only finitely many distinct multiplier ideals of the form $\mathcal{J}\left(X, \mathfrak{a}_{1}^{\lambda_{1}} \cdots \mathfrak{a}_{r}^{\lambda_{r}} \mathfrak{b}^{\beta}\right)$ for $\lambda_{1}+\cdots+\lambda_{r}=\alpha$. (This fact alone is not very hard to see from the definition of multiplier ideals.) In particular, the surjection $\mathcal{J}_{0} \rightarrow \mathcal{J}\left(X,\left(\mathfrak{a}_{1}+\cdots+\mathfrak{a}_{r}\right)^{\alpha} \mathfrak{b}^{\beta}\right)$ in our resolution implies the following (finite) summation formula; see also Section 4 for refinements.

Corollary 2. $\mathcal{J}\left(X,\left(\mathfrak{a}_{1}+\cdots+\mathfrak{a}_{r}\right)^{\alpha} \mathfrak{b}^{\beta}\right)=\sum_{\lambda_{1}+\cdots+\lambda_{r}=\alpha} \mathcal{J}\left(X, \mathfrak{a}_{1}^{\lambda_{1}} \cdots \mathfrak{a}_{r}^{\lambda_{r}} \mathfrak{b}^{\beta}\right)$.

Corollary 2 reduces the calculation of the multiplier ideals of arbitrary polynomial ideals to those of principal ideals. In the special case of a monomial ideal $\mathfrak{a}=\left\langle\mathbf{x}^{\gamma_{1}}, \ldots, \mathbf{x}^{\gamma_{r}}\right\rangle$, generated by the monomials in the polynomial ring $\mathbb{C}\left[x_{1}, \ldots, x_{d}\right]$ with exponent vectors $\gamma_{1}, \ldots, \gamma_{r} \in \mathbb{N}^{d}$, the summation formula becomes particularly explicit. For a subset $\Gamma \subseteq \mathbb{R}^{d}$, let conv $\Gamma$ denote its convex hull. By the integer part of a vector $\nu=\left(\nu_{1}, \ldots, \nu_{d}\right) \in \mathbb{R}^{d}$, we mean the vector $\left(\left\lfloor\nu_{1}\right\rfloor, \ldots,\left\lfloor\nu_{d}\right\rfloor\right) \in \mathbb{Z}^{d}$ whose entries are the greatest integers less than or equal to the coordinates of $\nu$.

Corollary 3. If $\mathfrak{a}=\left\langle\mathbf{x}^{\gamma_{1}}, \ldots, \mathbf{x}^{\gamma_{r}}\right\rangle$ is a monomial ideal in $\mathbb{C}\left[x_{1}, \ldots, x_{d}\right]$, then $\mathcal{J}\left(\mathfrak{a}^{\alpha}\right)$ is generated by the monomials in $\mathbb{C}\left[x_{1}, \ldots, x_{d}\right]$ whose exponent vectors are the integer parts of the vectors in $\operatorname{conv}\left\{\alpha \cdot \gamma_{1}, \ldots, \alpha \cdot \gamma_{r}\right\} \subseteq \mathbb{R}^{d}$.

Proof. Using Corollary 2 with $\mathfrak{a}_{j}=\left\langle\mathbf{x}^{\gamma_{j}}\right\rangle$, it suffices to note that the divisor of a single monomial has simple normal crossings, so no log resolution is necessary.

It is easy to check that for $\alpha=1$, the vectors in the conclusion of Corollary 3 are precisely those from Howald's result [How01], namely the vectors $\gamma \in \mathbb{N}^{d}$ such that $\gamma+(1, \ldots, 1)$ lies in the interior of the convex hull of all exponents of monomials in $\mathfrak{a}$.

Our approach to Theorem 1 is to construct a specific resolution satisfying the hypotheses, including the part about $\mathcal{J}_{0}$. The resolution we construct is cellular, in a sense generalizing the manner in which resolutions of monomial ideals can be cellular [BS98]; see [MS05, Chapter 4] for an introduction. In general, a complex in any abelian category could be called cellular if each homological degree is a direct sum indexed by the faces of a CW-complex, and the boundary maps are determined in a natural way from those of the CW-complex. An elementary way to phrase this in the present context is as follows. (Theorem 3.6 is more precise: a specific triangulation $\Delta$ is constructed in Section 2, and the sheaves $\mathcal{J}_{\sigma}$ are specified in Remark 3.3.) 
Theorem 4. Resume the notation from Theorem 1. There is a triangulation $\Delta$ of the simplex $\left\{\lambda \in \mathbb{R}^{r} \mid \sum_{i=1}^{r} \lambda_{i}=\alpha\right.$ and $\left.\lambda \geq 0\right\}$ such that

$$
\mathcal{J}_{i}=\bigoplus_{\sigma \in \Delta_{i}} \mathcal{J}_{\sigma}
$$

can be taken to be a direct sum indexed by the set $\Delta_{i}$ of $i$-dimensional faces $\sigma \in \Delta$, and the differential of $\mathcal{J}$. is induced by natural maps between ideal sheaves, using the signs from the boundary maps of $\Delta$. If $\lambda \in \Delta_{0}$ is a vertex, then $\mathcal{J}_{\lambda}=\mathcal{J}\left(X, \mathfrak{a}_{1}^{\lambda_{1}} \cdots \mathfrak{a}_{r}^{\lambda_{r}} \mathfrak{b}^{\beta}\right)$.

Comparing the final sentences of Theorems 1 and 4, a key point is that every possible multiplier ideal of the form $\mathcal{J}\left(X, \mathfrak{a}_{1}^{\lambda_{1}} \cdots \mathfrak{a}_{r}^{\lambda_{r}} \mathfrak{b}^{\beta}\right)$ occurs at some vertex $\lambda \in \Delta_{0}$. Writing down what it means for two such multiplier ideals to coincide, this stipulation provides strong hints as to potential choices for triangulations; see Section 2.

The proof of exactness for the cellular resolution in Theorem 4 proceeds by lifting the problem to an appropriate $\log$ resolution $X^{\prime} \rightarrow X$; see the proof of Theorem 3.6. Over $X^{\prime}$, we resolve the lifted ideal sheaf by a complex (of locally principal ideal sheaves in $\mathcal{O}_{X^{\prime}}$ ) that is, analytically locally at each point of $X^{\prime}$, a cellular free complex over a polynomial ring. This cellular free complex turns out to be a cellular free resolution of an appropriate monomial ideal; its construction and proof of acyclicity occupy Section 2, particularly Proposition 2.2. Having cellularly resolved the lifted sheaf over $X^{\prime}$, the desired cellular resolution over $\mathcal{O}_{X}$ is obtained by pushing forward to $X$ and using local vanishing; again, see the proof of Theorem 3.6. Thus Theorems 1 and 4 constitute a certain global version of cellular free resolutions of monomial ideals.

The acyclicity of the cellular free resolutions in Proposition 2.2 reduce to a simplicial homology vanishing statement, Corollary 1.7, for simplicial complexes obtained by deleting boundary faces from certain contractible manifolds-with-boundary. We deduce Corollary 1.7 from the following more general statement, which is of independent interest. Its rimmed hypothesis is satisfied by the barycentric subdivision of any polyhedral homology-manifold-with-boundary. In what follows, to delete a simplex $\sigma$ from a simplicial complex $M$ means to remove from $M$ every simplex containing $\sigma$.

Proposition 5. Fix a simplicial complex $M$ whose geometric realization $|M|$ is a homology-manifold with boundary $\partial M$. Assume that $M$ is rimmed, meaning that

$$
\text { if } \sigma \text { is a face of } M \text {, then } \sigma \cap|\partial M| \text { is a face of } M \text {. }
$$

Then deleting any collection of boundary simplices from $M$ results in a simplicial subcomplex whose (reduced) homology is canonically isomorphic to that of $M$.

\section{Combinatorial topological preliminaries}

In this section we collect some elementary results from simplicial topology. For additional background, see [Mun84], especially $\S 63$ for homology-manifolds, and [Zie95, Section 5.1] for polyhedral complexes. The reader interested solely in the construction of resolutions for Theorems 1 and 4, as opposed to their acyclicity proofs, can proceed to Section 2 after Example 1.1. The goal for the remainder of this section is the proof of Proposition 5 and its immediate consequence, Corollary 1.7.

Our convention is to identify any abstract simplicial complex $\Delta$ with the underlying topological space of any geometric realization $|\Delta|$. Thus, fixing a vertex set $V$, we view $\Delta$ as a collection of simplices (finite subsets of $V$ ), called faces of $\Delta$, such that 
any subset of any face of $\Delta$ is a face of $\Delta$. For example, we can express the link in $\Delta$ of a face $\sigma$ as the subcomplex

$$
\operatorname{link}_{\Delta}(\sigma)=\{\tau \in \Delta \mid \tau \cup \sigma \in \Delta \text { and } \tau \cap \sigma=\varnothing\} .
$$

In what follows, all links are taken inside of the ambient simplicial complex, unless otherwise noted. Our motivation is the following class of simplicial complexes.

Example 1.1. Let $\mathcal{P}$ be a polyhedral complex, such as a polyhedral subdivision of a polytope. (This example works just as well for any regular cell complex; see [Bjö84]). The barycentric subdivision of $\mathcal{P}$ is the simplicial complex $\Delta$ whose vertex set is the set of faces of $\mathcal{P}$, and whose simplices are the chains $P_{1}<\cdots<P_{\ell}$ of faces $P_{j} \in \mathcal{P}$. Here $P<Q$ means that $P$ is a proper face of $Q$. The barycentric subdivision $\Delta$ is homeomorphic to (the space underlying) $\mathcal{P}$; one way to realize $\Delta$ is to let each face $P_{1}<\cdots<P_{\ell}$ be the convex hull of the barycenters of the faces $P_{1}, \ldots, P_{\ell}$.

The main idea in the proof of Proposition 5 is to delete the boundary simplices one by one, in a suitable order, so that at each step the homology remains unchanged, using the following.

Lemma 1.2. Fix a simplicial complex $\Delta$ and a chain of simplicial subcomplexes

$$
\Delta=\Delta_{0} \supseteq \Delta_{1} \supseteq \cdots \supseteq \Delta_{r}=\Gamma .
$$

If the relative chain complex of each pair $\left(\Delta_{i-1}, \Delta_{i}\right)$ has vanishing homology for all $i=1, \ldots, r$, then the homology of $\Gamma$ is canonically isomorphic to that of $\Delta$.

Proof. Repeatedly apply the long exact sequence of homology.

Throughout the rest of this section, we use $M$ to denote a simplicial homologymanifold-with-boundary of dimension $\operatorname{dim}(M)=d$, defined as follows.

Definition 1.3. A simplicial homology-manifold-with-boundary of dimension $d$ is a simplicial complex whose maximal faces all have dimension $d$, and such that the link of each $i$-face has the homology of either a ball or a sphere of dimension $d-i-1$.

Remark 1.4. If the polyhedral complex $\mathcal{P}$ in Example 1.1 subdivides a homologymanifold-with-boundary, then the conditions of Proposition 5, including the rimmed condition (2), are satisfied by the barycentric subdivision of $\mathcal{P}$.

Lemma 1.5. The link of any $i$-face in $M$ is a dimension $d-i-1$ simplicial homologymanifold-with-boundary.

Proof. If $\sigma \subseteq M$ is a face of dimension $i$, then $\Gamma=\operatorname{link}_{M}(\sigma)$ is pure of dimension $d-i-1$ because $M$ is pure of dimension $d$. The condition on the homology of the link in $\Gamma$ of a face $\tau \in \Gamma$ holds simply because the condition holds for $\operatorname{link}_{M}(\sigma \cup \tau)$, which equals $\operatorname{link}_{\Gamma}(\tau)$ by definition.

The above lemma required no special hypotheses on $M$. Our final observation before the proof of Proposition 5 is that the condition (2) of being rimmed is inherited by links of boundary faces.

Lemma 1.6. Under the hypotheses of Proposition 5, the link of any boundary simplex of $M$ satisfies all of the hypotheses on $M$ in Proposition 5, including being rimmed. 
Proof. Let $\sigma$ be a boundary simplex of $M$. In view of Lemma 1.5, we need only show that $\operatorname{link}(\sigma)$ satisfies (2). This condition is a consequence of the equality

$$
\partial(\operatorname{link}(\sigma))=\partial M \cap \operatorname{link}(\sigma),
$$

because for any simplex $\tau$ in $\operatorname{link}(\sigma)$, the intersection $\tau \cap \partial(\operatorname{link}(\sigma))$ is forced to equal $\tau \cap \partial M$, which is a face of $\tau$ by (2).

To prove (3), we first show that if $\tau \in \partial(\operatorname{link}(\sigma))$ then $\tau \in \partial M$; note that for this implication, condition (2) is not necessary. Replacing $\tau$ by a face of $\partial(\operatorname{link}(\sigma))$ containing it, if necessary, we may assume that $\operatorname{dim}(\sigma \cup \tau)=d-1$; equivalently, $\tau$ is a maximal face of $\partial(\operatorname{link}(\sigma))$. In this case, there is only one maximal face of $\operatorname{link}(\sigma)$ containing $\tau$. But the faces of $\operatorname{link}(\sigma)$ containing $\tau$ are in bijection with the faces of $M$ containing $\sigma \cup \tau$. Hence there is only one maximal face of $M$ containing $\sigma \cup \tau$. Therefore $\sigma \cup \tau$ must be a boundary face of $M$. We conclude that $\tau \in \partial M$, because $\tau$ is a face of $\sigma \cup \tau$.

For the reverse containment, suppose now that $\tau \in \partial M \cap \operatorname{link}(\sigma)$, and let $\tau^{\prime}=$ $\tau \cup \sigma \in M$. The intersection $\tau^{\prime} \cap \partial M$ is a face of $M$ by condition (2), and it contains all of the vertices of $\sigma$ and $\tau$, because both $\sigma$ and $\tau$ are boundary faces of $M$. The only face of $\tau^{\prime}$ containing all of the vertices of $\sigma$ and $\tau$ is $\tau^{\prime}$ itself; hence $\tau^{\prime}=\tau^{\prime} \cap \partial M$ is a boundary face of $M$. It follows that $\operatorname{link}\left(\tau^{\prime}\right)$ has the homology of a ball (rather than a sphere). But $\operatorname{link}\left(\tau^{\prime}\right)=\operatorname{link}_{M}(\sigma \cup \tau)=\operatorname{link}_{\operatorname{link}(\sigma)}(\tau)$, so $\tau \in \partial(\operatorname{link}(\sigma))$.

Proof of Proposition 5. Use induction on the dimension of $M$. For $\operatorname{dim} M=1$ the statement is an elementary claim concerning subdivided intervals, so assume that $\operatorname{dim} M=d \geq 2$. Let $S$ be the collection of boundary faces to be deleted. Without loss of generality, we may assume that $S$ is a cocomplex inside $\partial M$, which means that if $\sigma \in S$ and $\tau \in \partial M$ such that $\tau \supseteq \sigma$, then $\tau \in S$.

Totally order the simplices in $S$ in such a way that all of the simplices of maximal dimension $d-1$ come first, then those of dimension $d-2$, and so on. Let $\sigma_{1}, \sigma_{2}, \ldots$ be this totally ordered sequence of simplices. Let $M_{i}$ be the result of deleting $\sigma_{1}, \ldots, \sigma_{i}$ from $M$, with $M=M_{0}$. The desired result will follow from Lemma 1.2, with $M=\Delta$, as soon as we show that the relative chain complexes $\mathcal{C} .\left(M_{i-1}, M_{i}\right)$ are all acyclic.

Let $\Gamma$ be the simplicial complex obtained by deleting the boundary from $\operatorname{link}_{M}\left(\sigma_{i}\right)$, which is acyclic by induction, via Lemma 1.6. We claim that $\mathcal{C} .\left(M_{i-1}, M_{i}\right)$ is (noncanonically) isomorphic to the reduced chain complex $\tilde{\mathcal{C}}$. $(\Gamma)$. Indeed, recall the bijective correspondence between the faces of $\operatorname{link}_{M}\left(\sigma_{i}\right)$ and the faces of $M$ containing $\sigma_{i}$. Under this correspondence, $\Gamma$ is mapped bijectively to the set

$$
\left\{\tau \in M \mid \tau \cap \partial M=\sigma_{i}\right\}=\left\{\tau \in M_{i-1} \mid \tau \supseteq \sigma_{i}\right\} .
$$

The faces of this cocomplex inside $M_{i-1}$ constitute a free basis of $\mathcal{C} .\left(M_{i-1}, M_{i}\right)$, and this induces an isomorphism from $\tilde{\mathcal{C}}$. $(\Gamma)$ to $\mathcal{C} .\left(M_{i-1}, M_{i}\right)$.

For future reference, here is the special case of Proposition 5 that we apply later.

Corollary 1.7. If $M$ is a contractible rimmed simplicial manifold-with-boundary, then deleting any collection of boundary faces from $M$ results in a simplicial subcomplex with vanishing reduced homology. 
Remark 1.8. It would be preferable to conclude in Corollary 1.7 that the subcomplex is contractible, and more generally that the subcomplex in Proposition 5 is homotopyequivalent to $M$, using [Bjö84, Lemma 10.3(i)]: if $\Delta_{i}=\Delta_{i-1} \cup \Gamma_{i}$ is a union of two simplicial subcomplexes such that $\Gamma_{i}$ and $\Delta_{i-1} \cap \Gamma_{i}$ are both contractible, then $\Delta_{i}$ is homotopy-equivalent to $\Delta_{i-1}$. The notation here is consistent with Lemma 1.2; in our case $\Delta_{i-1}$ is the result of deleting a boundary simplex $\sigma_{i}$ from $\Delta_{i}$, and $\Gamma_{i}$ is the cone from $\sigma_{i}$ over $\Delta_{i-1} \cap \Gamma_{i}$ (thus $\Gamma_{i}$ is the closed star of $\sigma_{i}$ in $\Delta_{i}$ ). The problem is that, while $\Gamma_{i}$ is always contractible (it is a cone), the subcomplex $\Delta_{i-1} \cap \Gamma_{i}=\operatorname{link}_{\Delta_{i}}\left(\sigma_{i}\right)$ need not be simply-connected, even though it has vanishing homology.

\section{A cellular free resolution}

Let $A \in \mathbb{Z}^{n \times r}$ be an integer matrix with $n$ rows and $r$ columns, and fix a real column vector $b \in \mathbb{R}^{n}$ with coordinates $b_{1}, \ldots, b_{n}$. (When applying these results in Section 3 , all entries in the matrix $A$ will be nonnegative.) Viewing the rows $A^{1}, \ldots, A^{n}$ as functionals on $\mathbb{R}^{r}$, we get an (infinite but locally finite) affine hyperplane arrangement

$$
\mathcal{A}=\bigcup_{\substack{z \in \mathbb{Z}^{n} \\ 1 \leq j \leq n}}\left\{\lambda \in \mathbb{R}^{r} \mid A^{j} \cdot \lambda+b_{j}=z_{j}\right\} .
$$

The arrangement $\mathcal{A}$ induces a polyhedral subdivision of $\mathbb{R}^{r}$. Fixing a nonnegative real number $\alpha \in \mathbb{R}_{\geq 0}$, the restriction of this polyhedral subdivision to the simplex

$$
\Delta^{\alpha}=\left\{\left(\lambda_{1}, \ldots, \lambda_{r}\right) \in \mathbb{R}_{\geq 0}^{r} \mid \lambda_{1}+\cdots+\lambda_{r}=\alpha\right\}
$$

is a polyhedral subdivision $\mathcal{A}_{\alpha}$ of $\Delta^{\alpha}$. Two points $\lambda$ and $\mu$ lie in the same (relatively open) cell of $\mathcal{A}_{\alpha}$ if and only if for each $j=1, \ldots, n$, there is an integer $z_{j}$ such that either

- $A^{j} \cdot \lambda+b_{j}=A^{j} \cdot \mu+b_{j}=z_{j}$, or else

- $A^{j} \cdot \lambda+b_{j}$ and $A^{j} \cdot \mu+b_{j}$ both lie in the open interval $\left(z_{j}, z_{j}+1\right)$.

For the duration of this paper, fix any polyhedral subdivision $\mathcal{P}$ of $\Delta^{\alpha}$ that refines $\mathcal{A}_{\alpha}$, meaning that every face of $\mathcal{P}$ is contained in a face of $\mathcal{A}_{\alpha}$. As in Example 1.1 , denote by $\Delta$ the barycentric subdivision of $\mathcal{P}$, realized so that the vertices of $\Delta$ are the barycenters of the cells of $\mathcal{P}$. We write $\sigma(P)$ for the vertex of $\Delta$ that is the barycenter of the polytope $P \in \mathcal{P}$.

Construct a module $M \subseteq \mathbb{C}\left[x_{1}^{ \pm 1}, \ldots, x_{n}^{ \pm 1}\right]$ generated by (Laurent) monomials over the polynomial ring $\mathbb{C}\left[x_{1}, \ldots, x_{n}\right]$, so $M$ is a Laurent monomial module [MS05, Definition 9.6], as follows. For each polytope $P \in \mathcal{P}$, set

$$
m_{P}=x_{1}^{\left\lfloor A^{1} \cdot \lambda+b_{1}\right\rfloor} \cdots x_{n}^{\left\lfloor A^{n} \cdot \lambda+b_{n}\right\rfloor} \text { for any } \lambda \in P^{\circ},
$$

where $\lfloor z\rfloor$ is the greatest integer less than or equal to $z$, and the cell $P^{\circ}$ is the relative interior of $P$; the monomial $m_{P}$ is well-defined because the greatest integer parts defining it are equal for all vectors in the same cell of $\mathcal{P}$. Now define

$$
M=\left\langle m_{P} \mid P \in \mathcal{P}\right\rangle .
$$

We can label each simplex in $\Delta$ with a monomial as follows. By virtue of the fact that each polytope in $\mathcal{P}$ indexes a monomial $m_{P}$ in the Laurent polynomial ring, the vertices of the simplicial complex $\Delta$, which are the barycenters $\sigma(P)$ of the 
polytopes $P \in \mathcal{P}$, are labeled with monomials. For each such vertex $\sigma(P)$, let us set $m_{\sigma(P)}=m_{P}$. More generally, for each simplex $\sigma \in \Delta$,

$$
\text { if } \sigma=\left(P_{1}<\cdots<P_{\ell}\right) \text { then set } m_{\sigma}=m_{P_{1}}
$$

i.e., we label $\sigma$ by the monomial of the smallest face in the chain. For (Laurent) monomials $m$ and $m^{\prime}$, we say that $m$ divides $m^{\prime}$ if $m^{\prime} / m$ is a monomial in $\mathbb{C}\left[x_{1}, \ldots, x_{n}\right]$.

Lemma 2.1. If $P<Q$ are polytopes in $\mathcal{P}$, then $m_{Q}$ divides $m_{P}$. Consequently, if $\sigma \in \Delta$ is a simplex, then $m_{\sigma}$ is the least common multiple of $\left\{m_{\tau} \mid \tau\right.$ is a vertex of $\left.\sigma\right\}$.

Proof. We prove only the first sentence, as the second follows easily. From the perspective of the greatest integer parts used in constructing $m_{P}$ and $m_{Q}$, the only difference between the barycenters of $P$ and $Q$ is that more of the affine functions $\lambda \mapsto A^{j} \cdot \lambda+b_{j}$ can take integer values on $\sigma(P)$ than on $\sigma(Q)$. In particular, if $\left\lfloor A^{j} \cdot \sigma(Q)+b_{j}\right\rfloor=z_{j}$, then $\left\lfloor A^{j} \cdot \sigma(P)+b_{j}\right\rfloor$ equals either $z_{j}$ or $z_{j}+1$. This being true for all $j$, we conclude that $m_{Q}$ divides $m_{P}$ (in fact, $m_{P} / m_{Q}$ is a squarefree monomial in $\mathbb{C}\left[x_{1}, \ldots, x_{n}\right]$ ).

Lemma 2.1 is precisely the condition under which the monomial labels $m_{\sigma}$ for $\sigma \in \Delta$ define a cellular free complex on $\Delta$ [BS98, Section 1] (see also Chapters 4 and 9 in [MS05] for background). Briefly, this is the complex

$$
0 \rightarrow \bigoplus_{\sigma \in \Delta_{r-1}}\left\langle m_{\sigma}\right\rangle \rightarrow \cdots \rightarrow \bigoplus_{\sigma \in \Delta_{i}}\left\langle m_{\sigma}\right\rangle \rightarrow \cdots \rightarrow \bigoplus_{\sigma \in \Delta_{0}}\left\langle m_{\sigma}\right\rangle \rightarrow 0
$$

of free $\mathbb{C}\left[x_{1}, \ldots, x_{n}\right]$-modules where the morphism $\left\langle m_{\sigma}\right\rangle \rightarrow\left\langle m_{\tau}\right\rangle$ of principal Laurent monomial modules is, for each codimension 1 face $\tau \subset \sigma$, the natural inclusion times \pm 1 . The sign is determined by arbitrary but fixed orientations for the simplices in $\Delta$.

Proposition 2.2. The cellular free complex supported on $\Delta$, in which $\sigma \in \Delta$ is labeled by $m_{\sigma}$, is a cellular free resolution of the Laurent monomial module $M$.

Proof. By [BS98, Proposition 1.2], we need to show that the simplicial subcomplex

$$
\Delta_{\preceq m}=\left\{\sigma \in \Delta \mid m_{\sigma} \text { divides } m\right\}
$$

is acyclic for all Laurent monomials $m$. Let $m=x_{1}^{e_{1}} \cdots x_{n}^{e_{n}}$. For $\sigma=\left(P_{1}<\cdots<P_{\ell}\right)$, the monomial $m_{\sigma}$ divides $m$ if and only if the barycenter $\tau=\sigma\left(P_{1}\right)$ satisfies the conditions $A^{j} \cdot \tau+b_{j}<e_{j}+1$ for all $j$.

Compare the subcomplex $\Gamma_{\preceq m} \subseteq \Delta$ consisting of those points $\lambda \in|\Delta|$ such that $A^{j} \cdot \lambda+b_{j} \leq e_{j}+1$ for all $j$. This subcomplex is a (compact convex) polytope, and hence $\Gamma_{\preceq m}$ is a contractible simplicial manifold-with-boundary. But $\Delta_{\preceq m}$ is obtained from $\Gamma_{\preceq m}$ by deleting those vertices $\tau$, necessarily on the boundary of $\Gamma_{\preceq m}$, having $A^{j} \cdot \tau+b_{j}=e_{j}+1$ for some $j$. The desired result is therefore a consequence of Corollary 1.7, which applies by Remark 1.4. 


\section{Log resolution to cellular resolution}

For the duration of this section, fix a normal $\mathbb{Q}$-Gorenstein complex variety $X$ and ideal sheaves $\mathfrak{a}_{1}, \ldots, \mathfrak{a}_{r}, \mathfrak{b}$ on $X$. In addition, fix a $\log$ resolution $\pi: X^{\prime} \rightarrow X$ of $\mathfrak{b}$ and $\mathfrak{a}_{i}+\mathfrak{a}_{j}$ for all $i, j \in\{1, \ldots, r\}$. By definition, this means that $\mathfrak{b} \cdot \mathcal{O}_{X^{\prime}}$ and $\left(\mathfrak{a}_{i}+\mathfrak{a}_{j}\right) \cdot \mathcal{O}_{X^{\prime}}$ are locally principal ideal sheaves on $X^{\prime}$. The special case $\mathfrak{a}_{i}=\mathfrak{a}_{j}$ implies that

$$
\begin{aligned}
\mathfrak{a}_{i} \cdot \mathcal{O}_{X^{\prime}} & =\mathcal{O}_{X^{\prime}}\left(-A_{i}\right) \text { for } i \in\{1, \ldots, r\} \text { and } \\
\mathfrak{b} \cdot \mathcal{O}_{X^{\prime}} & =\mathcal{O}_{X^{\prime}}(-B)
\end{aligned}
$$

are the ideal sheaves associated to effective divisors $A_{i}$ and $B$ on $X^{\prime}$.

We wish to calculate the multiplier ideals $\mathcal{J}\left(\left(\mathfrak{a}_{1}+\cdots+\mathfrak{a}_{r}\right)^{\alpha} \mathfrak{b}^{\beta}\right)$ for nonnegative real numbers $\alpha$ and $\beta$. This requires a $\log$ resolution of $\mathfrak{a}=\mathfrak{a}_{1}+\cdots+\mathfrak{a}_{r}$. Fortunately, the morphism $\pi: X^{\prime} \rightarrow X$ is one, as can be seen by applying the following locally on $X^{\prime}$.

Lemma 3.1. Let $R$ be a local integral domain.

1. If $\left\langle f_{1}, \ldots, f_{n}\right\rangle$ is a nonzero principal ideal of $R$, then there is an index $i \in\{1, \ldots, n\}$ such that $f_{i}$ divides $f_{j}$ for all $j \in\{1, \ldots, n\}$.

2. If $f_{1}, \ldots, f_{r} \in R$ are elements such that $\left\langle f_{i}, f_{j}\right\rangle$ is a principal ideal for all $i, j \in$ $\{1, \ldots, r\}$, then there exists a permutation $\xi \in S_{r}$ such that $f_{\xi(1)}\left|f_{\xi(2)}\right| \cdots \mid f_{\xi(r)}$. In particular $\left\langle f_{1}, \ldots, f_{r}\right\rangle=\left\langle f_{\xi(1)}\right\rangle$ is a principal ideal.

Proof. Suppose $\left\langle f_{1}, \ldots, f_{n}\right\rangle$ is generated by $f \in R$. Then each $f_{j}=f_{j}^{\prime} \cdot f$ is a multiple of $f$. On the other hand, $f=\sum c_{j} f_{j}=f \cdot \sum c_{j} f_{j}^{\prime}$ with $c_{j} \in R$. Since $R$ is a domain, this implies that $1=\sum c_{j} f_{j}^{\prime}$. Since $R$ is local, we conclude that some $f_{i}^{\prime}$ is a unit. This proves the first claim. The second is an immediate consequence of the first.

Similar to $\mathfrak{a}_{i}$ and $\mathfrak{b}$ from before, the ideal sheaf $\mathfrak{a} \cdot \mathcal{O}_{X^{\prime}}=\mathcal{O}_{X^{\prime}}(-C)$ is determined by an effective divisor $C$ on $X^{\prime}$. Let $D_{1}, \ldots, D_{n}$ be the set of prime divisors appearing in $A_{1}, \ldots, A_{r}, B, C$, and the relative canonical divisor $K_{X^{\prime} / X}$. Thus

$$
\begin{aligned}
A_{j} & =\sum_{i=1}^{n} a_{i j} D_{i} \text { for } j \in\{1, \ldots, r\}, \\
\beta B-K_{X^{\prime} / X} & =\sum_{i=1}^{n} b_{i} D_{i}, \text { and } \\
C & =\sum_{i=1}^{n} \gamma_{i} D_{i}
\end{aligned}
$$

in terms of $D_{1}, \ldots, D_{n}$, where the coefficients $a_{i j}$ and $\gamma_{i}$ are integers, but $b_{i}$ can be real numbers. Recall that the multiplier ideal of $\mathfrak{a}$ with weighting coefficient $\alpha$ is

$$
\mathcal{J}\left(\mathfrak{a}^{\alpha}\right)=\pi_{*} \mathcal{O}_{X^{\prime}}\left(-\left\lfloor\alpha C-K_{X^{\prime} / X}\right\rfloor\right) \subseteq \mathcal{O}_{X},
$$

where $\left\lfloor\sum_{i=1}^{n} \delta_{i} D_{i}\right\rfloor=\sum_{i=1}^{n}\left\lfloor\delta_{i}\right\rfloor D_{i}$ is the greatest integer divisor less than or equal to $\sum_{i=1}^{n} \delta_{i} D_{i}$. More generally, for $\lambda \in \mathbb{R}_{\geq 0}^{r}$ and $\beta \geq 0$, set

$$
\mathcal{J}\left(\mathfrak{a}_{1}^{\lambda_{1}} \cdots \mathfrak{a}_{r}^{\lambda_{r}} \mathfrak{b}^{\beta}\right)=\pi_{*} \mathcal{O}_{X^{\prime}}\left(-\left\lfloor\lambda_{1} A_{1}+\cdots+\lambda_{r} A_{r}+\beta B-K_{X^{\prime} / X}\right\rfloor\right) \subseteq \mathcal{O}_{X} .
$$


How do these multiplier ideals depend on the choices of $\lambda \in \mathbb{R}^{r}$ and $\beta \geq 0$ ? Given the decompositions into prime divisors $D_{1}, \ldots, D_{n}$ with integer coefficient matrix $A=\left(a_{i j}\right)$ and real vector $b=\left(b_{i}\right)$, we are verbatim in the situation from Section 2, whose notation we assume henceforth.

Lemma 3.2. For each polytope $P \in \mathcal{P}$, there is a single ideal sheaf $\mathcal{J}_{P} \subseteq \mathcal{O}_{X}$ that coincides with the multiplier ideals $\mathcal{J}\left(\mathfrak{a}_{1}^{\lambda_{1}} \cdots \mathfrak{a}_{r}^{\lambda_{r}} \mathfrak{b}^{\beta}\right)$ for all $\lambda \in P^{\circ}$ in the interior of $P$.

Proof. In fact, the greatest integer divisors $\left\lfloor\lambda_{1} A_{1}+\cdots+\lambda_{r} A_{r}+\beta B-K_{X^{\prime} / X}\right\rfloor$ on $X^{\prime}$ all coincide for $\lambda \in P^{\circ}$, by construction. Therefore we can define

$$
\mathcal{I}_{P}=\mathcal{O}_{X^{\prime}}\left(-\left\lfloor\lambda_{1} A_{1}+\cdots+\lambda_{r} A_{r}+\beta B-K_{X^{\prime} / X}\right\rfloor\right)
$$

for any $\lambda \in P^{\circ}$, and $\mathcal{J}_{P}=\pi_{*} \mathcal{I}_{P}$ does not depend on $\lambda \in P^{\circ}$.

As we did for monomials in Section 2 (before Lemma 2.1), having defined objects $\mathcal{I}_{P}$ and $\mathcal{J}_{P}$ indexed by polytopes in $\mathcal{P}$, we can define objects $\mathcal{I}_{\sigma}$ and $\mathcal{J}_{\sigma}$ indexed by simplices in $\Delta$ :

$$
\text { if } \sigma=\left(P_{1}<\cdots<P_{\ell}\right) \text { then set } \mathcal{I}_{\sigma}=\mathcal{I}_{P_{1}},
$$

and set $\mathcal{J}_{\sigma}=\pi_{*} \mathcal{I}_{\sigma}$.

Remark 3.3. Unwinding the definitions, we find that

$$
\mathcal{J}_{\sigma}=\mathcal{J}\left(\mathfrak{a}_{1}^{\lambda_{1}} \cdots \mathfrak{a}_{r}^{\lambda_{r}} \mathfrak{b}^{\beta}\right),
$$

where $\lambda$ is the barycenter of the smallest polytope $P_{1}$ in the chain $P_{1}<\cdots<P_{\ell}$ corresponding to the simplex $\sigma \in \Delta$. (Recall that $\Delta$ is the barycentric subdivision of the polyhedral complex $\mathcal{P}$ from the beginning of Section 2.)

The analogue of Lemma 2.1 holds here, as well.

Lemma 3.4. If $P<Q$ are polytopes in $\mathcal{P}$, then $\mathcal{I}_{Q} \supseteq \mathcal{I}_{P}$. Consequently, if $\sigma \in \Delta$, then $\mathcal{I}_{\sigma}=\bigcap\left\{\mathcal{I}_{\tau} \mid \tau\right.$ is a vertex of $\left.\sigma\right\}$. The same is true with $\mathcal{J}$ in place of $\mathcal{I}$.

Proof. The argument is essentially the same as the proof of Lemma 2.1.

Lemma 3.5. Resume the notation involving $\mathfrak{a}=\mathfrak{a}_{1}+\cdots+\mathfrak{a}_{r}$ from after Lemma 3.1, so $\mathfrak{a} \cdot \mathcal{O}_{X^{\prime}}=\mathcal{O}_{X^{\prime}}(-C)$. Then, as ideal sheaves over $X^{\prime}$,

$$
\mathcal{O}_{X^{\prime}}\left(-\left\lfloor\alpha C+\beta B-K_{X^{\prime} / X}\right\rfloor\right)=\sum_{P \in \mathcal{P}} \mathcal{I}_{P} .
$$

Proof. Let $p \in X^{\prime}$ be an arbitrary point. By Lemma 3.1, there is an open neighborhood $U$ of $p$ and a permutation $\xi \in S_{r}$ such that after restricting to $U$, we have $A_{\xi(1)} \preceq \cdots \preceq A_{\xi(r)}$, where $E \preceq F$ for divisors $E$ and $F$ means that $F-E$ is effective. It follows that on $U$ we have $C=A_{\xi(1)}$, and also

$$
\alpha A_{\xi(1)} \preceq \lambda_{1} A_{1}+\cdots+\lambda_{r} A_{r}
$$

whenever $\lambda_{1}+\ldots+\lambda_{r}=\alpha$. Hence both sides of the desired equality are equal to $\mathcal{O}_{X^{\prime}}\left(-\left\lfloor\alpha A_{\xi(1)}+\beta B-K_{X^{\prime} / X}\right\rfloor\right)$ on $U$. Equality holds on $X^{\prime}$ because $p$ is arbitrary. 
We have arrived at our main result. For notational purposes, choose an arbitrary but fixed collection of orientations for the simplices in $\Delta$. There results an incidence function that assigns to each codimension 1 face $\tau$ of each simplex $\sigma$ a $\operatorname{sign}(-1)^{\sigma, \tau}$.

Theorem 3.6. With $\mathcal{J}_{\sigma}$ as in Remark 3.3, there is an exact sequence

$$
0 \rightarrow \bigoplus_{\sigma \in \Delta_{r-1}} \mathcal{J}_{\sigma} \rightarrow \cdots \rightarrow \bigoplus_{\sigma \in \Delta_{i}} \mathcal{J}_{\sigma} \rightarrow \cdots \rightarrow \bigoplus_{\sigma \in \Delta_{0}} \mathcal{J}_{\sigma} \rightarrow \mathcal{J}\left(\left(\mathfrak{a}_{1}+\cdots+\mathfrak{a}_{r}\right)^{\alpha} \mathfrak{b}^{\beta}\right) \rightarrow 0
$$

of sheaves on $X$, in which the morphism $\mathcal{J}_{\sigma} \rightarrow \mathcal{J}_{\tau}$ is inclusion times $(-1)^{\sigma, \tau}$. Here, $\Delta_{i}=\{\sigma \in \Delta \mid \operatorname{dim}(\sigma)=i\}$ is the set of $i$-dimensional simplices in $\Delta$.

Proof. We shall first verify the exactness of the complex

$$
0 \rightarrow \bigoplus_{\sigma \in \Delta_{r-1}} \mathcal{I}_{\sigma} \rightarrow \cdots \rightarrow \bigoplus_{\sigma \in \Delta_{i}} \mathcal{I}_{\sigma} \rightarrow \cdots \rightarrow \bigoplus_{\sigma \in \Delta_{0}} \mathcal{I}_{\sigma} \rightarrow \sum_{\sigma \in \Delta_{0}} \mathcal{I}_{\sigma} \rightarrow 0
$$

on $X^{\prime}$, where it should be noted that the sum at the far right is not a direct sum. It is enough to verify exactness at the stalk of an arbitrary point $p \in X^{\prime}$. Reordering the prime divisors $D_{1}, \ldots, D_{n}$ appearing in $A_{1}, \ldots, A_{r}, B$, and $K_{X^{\prime} / X}$ if necessary, we assume that only $D_{1}, \ldots, D_{k}$ pass through $p$. Since $D_{1}, \ldots, D_{n}$ intersect transversally, if we pass to an analytic neighborhood or completion at $p$, then we can choose local coordinates $x_{1}, \ldots, x_{k}$ so that $D_{i}$ is given by the vanishing of $x_{i}$ for $i=1, \ldots, k$. In these coordinates, $\mathcal{I}_{\sigma}$ becomes the principal Laurent monomial module in $\mathbb{C}\left[x_{1}^{ \pm 1}, \ldots, x_{k}^{ \pm 1}\right]$ generated by the monomial $m_{\sigma}$ from before Lemma 2.1, except that all of the variables $x_{k+1}, \ldots, x_{n}$ have been set equal to 1 in $m_{\sigma}$. The exactness thus follows from Proposition 2.2, given that our polyhedral complex $\mathcal{P}$ refines the subdivision induced by the linear functionals $A^{j} \cdot \lambda+b_{j}$ for $j=1, \ldots, k$, instead of $j=1, \ldots, n$. (Another reason the exactness follows from Proposition 2.2 is that setting the variables equal to 1 is the exact operation of "homogeneous localization"; see [Mil00, Section 3.6], particularly Proposition 3.31.2 there.)

Pushing forward under $\pi$ completes the proof. Indeed, Lemma 3.5 implies that

$$
\sum_{\sigma \in \Delta_{0}} \mathcal{I}_{\sigma}=\sum_{P \in \mathcal{P}} \mathcal{I}_{P}=\mathcal{O}_{X^{\prime}}\left(-\left\lfloor\alpha C+\beta B-K_{X^{\prime} / X}\right\rfloor\right)
$$

pushes forward to yield $\mathcal{J}\left(\left(\mathfrak{a}_{1}+\cdots+\mathfrak{a}_{r}\right)^{\alpha} \mathfrak{b}^{\beta}\right)$, while local vanishing [Laz04, Theorem 9.4.17(i)] guarantees that the higher direct images vanish on all $\mathcal{I}_{\sigma}$.

Remark 3.7. In the special case where $X=\operatorname{Spec} \mathbb{C}\left[x_{1}, \ldots, x_{n}\right]$ and $\mathfrak{a}_{1}, \ldots, \mathfrak{a}_{r}, \mathfrak{b}$ are all principal monomial ideals, all of the $\mathcal{J}_{\sigma}$ are principal monomial ideals as well, so the exact sequence in Theorem 3.6 becomes a cellular resolution of the monomial ideal $\mathcal{J}\left(\left(\mathfrak{a}_{1}+\cdots+\mathfrak{a}_{r}\right)^{\alpha} \mathfrak{b}^{\beta}\right)$ in the sense of [MS05, Definition 4.3].

Example 3.8. Let us illustrate Theorem 3.6 by an example. Take $X=\operatorname{Spec} \mathbb{C}[x, y]$, $r=\alpha=2, \mathfrak{a}_{1}=\langle x y\rangle, \mathfrak{a}_{2}=\langle x+y\rangle$, and $\mathfrak{b}=\mathcal{O}_{X}$. A direct computation shows that 
on the doubled 1-simplex $\left\{\left(\lambda_{1}, \lambda_{2}\right) \in \mathbb{R}_{\geq 0} \mid \lambda_{1}+\lambda_{2}=2\right\}$, we have

$$
\mathcal{J}\left(\mathfrak{a}_{1}^{\lambda_{1}} \mathfrak{a}_{2}^{\lambda_{2}}\right)= \begin{cases}\left\langle x^{2} y^{2}\right\rangle & \text { if } \lambda_{2}=0 \\ \langle x y\rangle & \text { if } 0<\lambda_{2}<1 \\ \langle x y(x+y)\rangle & \text { if } \lambda_{2}=1 \\ \langle x+y\rangle & \text { if } 1<\lambda_{2}<2 \\ \left\langle(x+y)^{2}\right\rangle & \text { if } \lambda_{2}=2 .\end{cases}
$$

These regions where $\mathcal{J}\left(\mathfrak{a}_{1}^{\lambda_{1}} \mathfrak{a}_{2}^{\lambda_{2}}\right)$ stays constant yield a polyhedral subdivision $\mathcal{P}$ of the doubled 1-simplex, and we let $\Delta$ be the barycentric subdivision of $\mathcal{P}$. Explicitly, $\Delta$ consists of five vertices

$$
v_{1}=(2,0), v_{2}=\left(\frac{3}{2}, \frac{1}{2}\right), v_{3}=(1,1), v_{4}=\left(\frac{1}{2}, \frac{3}{2}\right), v_{5}=(0,2)
$$

and four edges

$$
l_{i}=\text { the line segment between } v_{i} \text { and } v_{i+1}, \text { for } i=1,2,3,4 .
$$

The vertices in $\Delta$ are naturally labeled with the multiplier ideals

$$
\begin{aligned}
\mathcal{J}_{v_{1}} & =\mathcal{J}\left(\mathfrak{a}_{1}^{2} \mathfrak{a}_{2}^{0}\right)=\left\langle x^{2} y^{2}\right\rangle, \\
\mathcal{J}_{v_{2}} & =\mathcal{J}\left(\mathfrak{a}_{1}^{3 / 2} \mathfrak{a}_{2}^{1 / 2}\right)=\langle x y\rangle, \\
\mathcal{J}_{v_{3}} & =\mathcal{J}\left(\mathfrak{a}_{1}^{1} \mathfrak{a}_{2}^{1}\right)=\langle x y(x+y)\rangle, \\
\mathcal{J}_{v_{4}} & =\mathcal{J}\left(\mathfrak{a}_{1}^{1 / 2} \mathfrak{a}_{2}^{3 / 2}\right)=\langle x+y\rangle, \\
\mathcal{J}_{v_{5}} & =\mathcal{J}\left(\mathfrak{a}_{1}^{0} \mathfrak{a}_{2}^{2}\right)=\left\langle(x+y)^{2}\right\rangle .
\end{aligned}
$$

The edges of $\Delta$ are labeled by $\mathcal{J}_{l_{i}}$, which for $i=1,2,3,4$ is defined to be the least common multiple of $\mathcal{J}_{v_{i}}$ and $\mathcal{J}_{v_{i+1}}$. It is easily verified that $\mathcal{J}_{l_{i}}$ equals the smaller one of $\mathcal{J}_{v_{i}}$ and $\mathcal{J}_{v_{i+1}}$. The exact sequence of Theorem 3.6 is

$$
0 \rightarrow \bigoplus_{i=1}^{4} \mathcal{J}_{l_{i}} \rightarrow \bigoplus_{i=1}^{5} \mathcal{J}_{v_{i}} \rightarrow \mathcal{J}\left(\left(\mathfrak{a}_{1}+\mathfrak{a}_{2}\right)^{2}\right) \rightarrow 0
$$

or more explicitly,

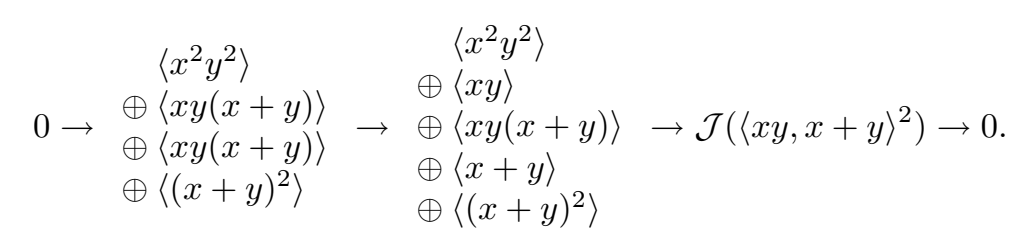

After canceling redundant terms, this is essentially a Koszul resolution

$$
0 \rightarrow\langle x y(x+y)\rangle \rightarrow\langle x y\rangle \oplus\langle x+y\rangle \rightarrow \mathcal{J}\left(\langle x y, x+y\rangle^{2}\right) \rightarrow 0 .
$$




\section{Applications}

The Introduction already contains some immediate applications of Theorem 3.6, namely the Takagi-style summation formula in Corollary 2 and the derivation of Howald's monomial multiplier ideal formula in Corollary 3. In this section, we collect further applications: a new exactness proof for the Skoda complex, and additional summation formulas for multiplier ideals, including the graded system case.

We begin with the summation formulas. First, we note the following.

Remark 4.1. Theorem 3.6 still holds when $\mathfrak{b}^{\beta}$ is replaced by $\mathfrak{b}_{1}^{\beta_{1}} \cdots \mathfrak{b}_{s}^{\beta_{s}}$, since the contants $\beta_{k}$ merely translate the affine hyperplane arrangement $\mathcal{A}$ in Section 2 .

Corollary 4.2. Let $\mathfrak{a}_{1}, \ldots, \mathfrak{a}_{r}, \mathfrak{b}_{1}, \ldots, \mathfrak{b}_{s} \subseteq \mathcal{O}_{X}$ be nonzero ideal sheaves on a normal $\mathbb{Q}$-Gorenstein variety $X$ and let $\alpha, \beta_{1}, \ldots, \beta_{s}$ be positive real numbers. Then

$$
\mathcal{J}\left(X,\left(\mathfrak{a}_{1}+\cdots+\mathfrak{a}_{r}\right)^{\alpha} \mathfrak{b}_{1}^{\beta_{1}} \cdots \mathfrak{b}_{s}^{\beta_{s}}\right)=\sum_{\lambda_{1}+\cdots+\lambda_{r}=\alpha} \mathcal{J}\left(X, \mathfrak{a}_{1}^{\lambda_{1}} \cdots \mathfrak{a}_{r}^{\lambda_{r}} \mathfrak{b}_{1}^{\beta_{1}} \cdots \mathfrak{b}_{s}^{\beta_{s}}\right)
$$

Proof. This follows immediately from the surjectivity on the right of the exact sequence in Theorem 3.6.

Our next result concerns the notion of graded system of ideals. For the definition of this and the multiplier ideal associated to it, see [Laz04, Section 11.1.B]. A special case has already appeared in [Tak05, Proposition 4.10].

Corollary 4.3. Corollary 4.2 still holds when the ideal sheaves $\mathfrak{a}_{i}$ and $\mathfrak{b}_{j}$ are all replaced by graded systems of ideals.

Proof. The formula for $r>2$ can be obtained by repeatedly applying the formula for $r=2$, so it suffices to prove this case, i.e.,

$$
\mathcal{J}\left(X,\left(\mathfrak{a} .+\mathfrak{b}_{\bullet}\right)^{\alpha} \mathfrak{c}_{1, \bullet}^{\beta_{1}} \cdots \mathfrak{c}_{s, \bullet}^{\beta_{s}}\right)=\sum_{0 \leq t \leq \alpha} \mathcal{J}\left(X, \mathfrak{a}_{\bullet}^{\alpha-t} \mathfrak{b}_{\bullet}^{t} \cdot \mathfrak{c}_{1, \bullet}^{\beta_{1}} \cdots \mathfrak{c}_{s, \bullet}^{\beta_{s}}\right)
$$

First let us verify $\supseteq$, i.e., $\mathcal{J}\left(X, \mathfrak{a}_{\bullet}^{\alpha-t} \mathfrak{b}_{\bullet}^{t} \cdot \mathfrak{c}_{1, \bullet}^{\beta_{1}} \cdots \mathfrak{c}_{s, \bullet}^{\beta_{s}}\right) \subseteq \mathcal{J}\left(X,\left(\mathfrak{a} \cdot+\mathfrak{b}_{\bullet}\right)^{\alpha} \mathfrak{c}_{1, \bullet}^{\beta_{1}} \cdots \mathfrak{c}_{s, \bullet}^{\beta_{s}}\right)$ for any fixed $t \in[0, \alpha]$. By definition, the left-hand side is equal to $\mathcal{J}\left(X, \mathfrak{a}_{p}^{\frac{\alpha-t}{p}} \mathfrak{b}_{p}^{\frac{t}{p}} \cdot \mathfrak{c}_{1, p}^{\frac{\beta_{1}}{p}} \cdots \mathfrak{c}_{s, p}^{\frac{\beta_{s}}{p}}\right)$ for some large $p$, and we see that

$$
\begin{aligned}
& \mathcal{J}\left(X, \mathfrak{a}_{p}^{\frac{\alpha-t}{p}} \mathfrak{b}_{p}^{\frac{t}{p}} \cdot \mathfrak{c}_{1, p}^{\frac{\beta_{1}}{p}} \cdots \mathfrak{c}_{s, p}^{\frac{\beta_{s}}{p}}\right) \subseteq \mathcal{J}\left(X,\left(\mathfrak{a}_{p}+\mathfrak{b}_{p}\right)^{\frac{\alpha}{p}} \mathfrak{c}_{1, p}^{\frac{\beta_{1}}{p}} \cdots \mathfrak{c}_{s, p}^{\frac{\beta_{s}}{p}}\right) \quad \text { (by Corollary 4.2) } \\
& \subseteq \mathcal{J}\left(X,\left(\mathfrak{a} .+\mathfrak{b}_{.}\right)_{p}^{\frac{\alpha}{p}} \mathfrak{c}_{1, p}^{\frac{\beta_{1}}{p}} \cdots \mathfrak{c}_{s, p}^{\frac{\beta_{s}}{p}}\right) \\
& \subseteq \mathcal{J}\left(X,\left(\mathfrak{a} .+\mathfrak{b}_{\bullet}\right)^{\alpha} \mathfrak{c}_{1, \bullet}^{\beta_{1}} \cdots \mathfrak{c}_{s, \bullet}^{\beta_{s}}\right) \quad \text { (by definition). }
\end{aligned}
$$

To prove the reverse inclusion, first by definition there exists some large $p$ such that

$$
\mathcal{J}\left(X,\left(\mathfrak{a} \cdot+\mathfrak{b}_{\bullet}\right)^{\alpha} \mathfrak{c}_{1, \bullet}^{\beta_{1}} \cdots \mathfrak{c}_{s, \bullet}^{\beta_{s}}\right)=\mathcal{J}\left(X,\left(\sum_{i=0}^{p} \mathfrak{a}_{i} \mathfrak{b}_{p-i}\right)^{\frac{\alpha}{p}} \mathfrak{c}_{1, p}^{\frac{\beta_{1}}{p}} \cdots \mathfrak{c}_{s, p}^{\frac{\beta_{s}}{p}}\right)
$$


and by Corollary 4.2 this right-hand side can be expressed as

$$
\sum_{\lambda_{0}+\cdots+\lambda_{p}=\frac{\alpha}{p}} \mathcal{J}\left(X,\left(\prod_{i=1}^{p} \mathfrak{a}_{i}^{\lambda_{i}}\right)\left(\prod_{i=0}^{p-1} \mathfrak{b}_{p-i}^{\lambda_{i}}\right) \cdot \mathfrak{c}_{1, p}^{\frac{\beta_{1}}{p}} \cdots \mathfrak{c}_{s, p}^{\frac{\beta_{s}}{p}}\right) .
$$

Now take a positive integer $m$ which is divisible by $1,2, \ldots, p$. Since $\mathfrak{a}_{\bullet}, \mathfrak{b}$. and $\mathfrak{c}_{j,}$. are graded systems, we have

$$
\mathfrak{a}_{i}^{\lambda_{i}}=\mathfrak{a}_{i}^{\frac{m}{i} \frac{i \lambda_{i}}{m}} \subseteq \mathfrak{a}_{m}^{\frac{i \lambda_{i}}{m}}, \quad \mathfrak{b}_{p-i}^{\lambda_{i}}=\mathfrak{b}_{p-i}^{\frac{m}{p-i}} \frac{(p-i) \lambda_{i}}{m} \subseteq \mathfrak{b}_{m}^{\frac{(p-i) \lambda_{i}}{m}}, \quad \mathfrak{c}_{j, p}^{\frac{\beta_{j}}{p}}=\mathfrak{c}_{j, p}^{\frac{m}{p} \frac{\beta_{j}}{m}} \subseteq \mathfrak{c}_{j, m}^{\frac{\beta_{j}}{m}},
$$

so

$$
\left(\prod_{i=1}^{p} \mathfrak{a}_{i}^{\lambda_{i}}\right)\left(\prod_{i=0}^{p-1} \mathfrak{b}_{p-i}^{\lambda_{i}}\right) \cdot \mathfrak{c}_{1, p}^{\frac{\beta_{1}}{p}} \cdots \mathfrak{c}_{s, p}^{\frac{\beta_{s}}{p}} \subseteq \sum_{\mathfrak{i}}^{p} \frac{p}{m} \frac{i \lambda_{i}}{m} \mathfrak{b}_{m}^{p=0} \frac{(p-i) \lambda_{i}}{m} \cdot \mathfrak{c}_{1, m}^{\frac{\beta_{1}}{m}} \cdots \mathfrak{c}_{s, m}^{\frac{\beta_{s}}{m}},
$$

and since $\lambda_{0}+\cdots+\lambda_{p}=\frac{\alpha}{p}$, if we let $t:=\sum_{i=0}^{p-1}(p-i) \lambda_{i}$, then $\sum_{i=1}^{p} i \lambda_{i}=\alpha-t$, hence

$$
\begin{aligned}
\mathcal{J}\left(X,\left(\prod_{i=1}^{p} \mathfrak{a}_{i}^{\lambda_{i}}\right)\left(\prod_{i=0}^{p-1} \mathfrak{b}_{p-i}^{\lambda_{i}}\right) \cdot \mathfrak{c}_{1, p}^{\frac{\beta_{1}}{p}} \cdots \mathfrak{c}_{s, p}^{\frac{\beta_{s}}{p}}\right) & \subseteq \mathcal{J}\left(X, \mathfrak{a}_{m}^{\frac{\alpha-t}{m}} \mathfrak{b}_{m}^{\frac{t}{m}} \cdot \mathfrak{c}_{1, m}^{\frac{\beta_{1}}{m}} \cdots \mathfrak{c}_{s, m}^{\frac{\beta_{s}}{m}}\right) \\
& \subseteq \mathcal{J}\left(X, \mathfrak{a}_{\bullet}^{\alpha-t} \mathfrak{b}_{\bullet}^{t} \cdot \mathfrak{c}_{1, \bullet}^{\beta_{1}} \cdots \mathfrak{c}_{s, \bullet}^{\beta_{s}}\right) .
\end{aligned}
$$

All of the corollaries of Theorem 3.6 we have given so far are Takagi-style summation formulas which may also be obtained by straightforwardly generalizing the proof of Takagi's original formula (1) (see [Tak05, Theorem 3.2]). However, in our final application we want to demonstrate that Theorem 3.6 may be potentially more useful than (1) by deriving the exactness of the Skoda complex from the former. For this we assume that the ideal sheaves $\mathfrak{a}_{1}, \ldots, \mathfrak{a}_{r}$ are locally principal on the $\mathbb{Q}$-Gorenstein variety $X$. For every nonnegative integer $\alpha<r$, the inclusion $\mathfrak{a}_{i} \rightarrow \mathcal{O}_{X}$ induces a natural map $\mathfrak{a}_{i} \mathcal{J}\left(\mathfrak{a}^{\alpha} \mathfrak{b}^{\beta}\right) \rightarrow \mathcal{J}\left(\mathfrak{a}^{\alpha+1} \mathfrak{b}^{\beta}\right)$. Writing $\mathfrak{a}_{\tau}=\prod_{i \in \tau} \mathfrak{a}_{i}$ for $\tau \in\{0,1\}^{r} \subseteq \mathbb{R}^{r}$, the Skoda complex is the cellular complex

$0 \rightarrow \mathfrak{a}_{1} \cdots \mathfrak{a}_{r} \mathcal{J}\left(\mathfrak{b}^{\beta}\right) \rightarrow \cdots \rightarrow \bigoplus_{|\tau|=r-\alpha} \mathfrak{a}_{\tau} \mathcal{J}\left(\mathfrak{a}^{\alpha} \mathfrak{b}^{\beta}\right) \rightarrow \cdots \rightarrow \bigoplus_{i=1}^{r} \mathfrak{a}_{i} \mathcal{J}\left(\mathfrak{a}^{r-1} \mathfrak{b}^{\beta}\right) \rightarrow \mathcal{J}\left(\mathfrak{a}^{r} \mathfrak{b}^{\beta}\right) \rightarrow 0$

supported on a simplex whose faces are in bijection with the vectors $\tau \in\{0,1\}^{r}$.

Corollary 4.4. The Skoda complex is exact.

A proof for smooth $X$ appears in [Laz04, Section 9.6.C]. Here, we provide an alternate proof, which works with no extra effort in the $\mathbb{Q}$-Gorenstein setting.

Proof. Consider the subdivisions $\mathcal{A}_{\alpha}$ of the simplices $\Delta^{\alpha}$ for $\alpha=0, \ldots, r$, defined at the beginning of Section 2. Every vector $\tau \in\{0,1\}^{r} \subseteq \mathbb{R}^{r}$ induces an embedding $\Delta^{\alpha} \hookrightarrow \Delta^{r}$, where $\alpha=r-|\tau|$, by adding $\tau$. Choose a refinement $\mathcal{P}$ of $\mathcal{A}_{r}$ so fine that the subdivision induced on $\Delta^{\alpha}$ under every one of these inclusions, for all $\tau$ and all 
$\alpha=0, \ldots, r$, refines $\mathcal{A}_{\alpha}$. For example, in the notation from the beginning of Section 2 , let $\mathcal{P}$ be the subdivision induced by the affine hyperplane arrangement

$$
\mathcal{A}+\{0,1\}^{r}=\bigcup_{\substack{z \in \mathbb{Z}^{n} \\ 1 \leq j \leq n}} \bigcup_{\tau \in\{0,1\}^{r}}\left\{\lambda+\tau \in \mathbb{R}^{r} \mid A^{j} \cdot \lambda+b_{j}=z_{j}\right\}
$$

obtained by translating the arrangement $\mathcal{A}$ up by every vector $\tau \in\{0,1\}^{r}$.

As in Theorem 3.6, let $\Delta$ be the barycentric subdivision of $\mathcal{P}$. Recall the definition of $\mathcal{J}_{\sigma}=\mathcal{J}\left(\mathfrak{a}_{1}^{\lambda_{1}} \cdots \mathfrak{a}_{r}^{\lambda_{r}} \mathfrak{b}^{\beta}\right)$ from Remark 3.3, and write $\lambda(\sigma)=\lambda=\left(\lambda_{1}, \ldots, \lambda_{r}\right)$. Define a double complex $\mathcal{J}_{., \text {, }}$ in which

$$
\mathcal{J}_{p, q}=\bigoplus_{\operatorname{dim}(\sigma)=q} \bigoplus_{\substack{|\tau|=p \\ \tau \preceq \lambda(\sigma)}} \mathcal{J}_{\sigma}
$$

where $\tau \preceq \lambda(\sigma)$ means that $\lambda(\sigma)-\tau \in \Delta^{r-|\tau|}$ (equivalently, $\lambda(\sigma)-\tau$ has nonnegative entries). Each horizontal complex $\mathcal{J}_{\bullet, q}$ is a direct sum, over $\sigma \in \Delta$ with $\operatorname{dim}(\sigma)=q$, of complexes $\mathcal{J}_{\sigma} \otimes_{\mathbb{C}} \tilde{C}$. $\left(\Gamma_{\tau}\right)$, where $\tau \preceq \lambda(\sigma)$ is maximal and $\tilde{C}$. $\left(\Gamma_{\tau}\right)$ is the reduced chain complex of a simplex $\Gamma_{\tau}$ with $|\tau|$ many vertices. The vertical differentials of $\mathcal{J}_{\bullet,}$. are induced by the differentials of Theorem 3.6, for each fixed $\tau$. In particular, the vertical complex on the summands $\mathcal{J}_{\sigma}$ indexed by a fixed $\tau \preceq \lambda=\lambda(\sigma)$ is a resolution of $\mathfrak{a}_{\tau} \mathcal{J}\left(\mathfrak{a}^{r-|\tau|} \mathfrak{b}^{\beta}\right)$ by Theorem 3.6, because

$$
\mathcal{J}\left(\mathfrak{a}_{1}^{\lambda_{1}} \cdots \mathfrak{a}_{r}^{\lambda_{r}} \mathfrak{b}^{\beta}\right)=\mathfrak{a}_{\tau} \mathcal{J}\left(\mathfrak{a}_{1}^{\lambda_{1}-\tau_{1}} \cdots \mathfrak{a}_{r}^{\lambda_{r}-\tau_{r}} \mathfrak{b}^{\beta}\right) .
$$

It follows that the vertical homology of $\mathcal{J}_{\bullet,}$. is the Skoda complex. On the other hand, the horizontal homology of $\mathcal{J}_{.,}$. is identically zero because every simplex $\Gamma_{\tau}$ is contractible. The standard spectral sequence argument shows the desired result.

\section{Acknowledgements}

We are grateful to Rob Lazarsfeld for valuable discussions, and to Shunsuke Takagi for the suggestion to work with singular varieties. EM was supported by NSF CAREER grant DMS-0449102 and a University of Minnesota McKnight Land-Grant Professorship. The latter funded his visit to the University of Michigan, which he wishes to thank for its hospitality while this work was completed.

\section{References}

[BS98] D. Bayer and B. Sturmfels, Cellular resolutions of monomial modules, J. Reine Angew. Math. 502 (1998), 123-140.

[Bjö84] A. Björner, Topological methods, Handbook of combinatorics, Vol. 1, 2, 1819-1872, Elsevier, Amsterdam, 1995.

[DEL00] J.-P. Demailly, L. Ein and R. Lazarsfeld, A subadditivity property of multiplier ideals, Michigan Math. J. 48 (2000), 137-156.

[How01] J. Howald, Multiplier ideals of monomial ideals, Trans. Amer. Math. Soc. 353 (2001), no. 7, 2665-2671 (electronic).

[Laz04] R. Lazarsfeld, Positivity in Algebraic Geometry I-II, Ergeb. Math. Grenzgeb., vols. 48-49, Berlin: Springer, 2004.

[Mil00] E. Miller, The Alexander duality functors and local duality with monomial support, J. Algebra 231 (2000), 180-234.

[MS05] E. Miller and B. Sturmfels, Combinatorial commutative algebra, Graduate Texts in Mathematics 227, New York: Springer, 2005. 
[Mun84] J. R. Munkres, Elements of algebraic topology, Addison-Wesley, Menlo Park, CA, 1984.

[Mus02] M. Mustaţă, The multiplier ideals of a sum of ideals, Trans. Amer. Math. Soc. 354 (2002), no. $1,205-217$.

[Tak05] S. Takagi, Formulas for multiplier ideals on singular varieties, Amer. J. Math. 128 (2006), 1345-1362. arXiv:math.AG/0410612

[Zie95] G. Ziegler, Lectures on polytopes, Graduate Texts in Mathematics Vol. 152, Springer-Verlag, New York, 1995.

Department of Mathematics, University of Michigan, Ann Arbor, Mi 48109-1043

E-mail address, Shin-Yao Jow: syjow@umich.edu

School of Mathematics, University of Minnesota, Minneapolis, MN 55455

E-mail address, Ezra Miller: ezra@math.umn.edu 